\title{
EFFECTS OF THE USE OF CIGARETTES ON THE FUNCTION OF FRONTAL LOBE IN MIDDLE AND UPPER MIDDLE-AGED JAPANESE ADULTS
}

\author{
Takeshi HATTA $^{1 \text {, }}$, Miyuki OKUMURA ${ }^{1}$, Naoko NAGAHARA ${ }^{1)}$, Emi ITO $^{1)}$, \\ ${ }^{1)}$ Nagoya University, Japan \\ Yoshinori ITO $^{2)}$ and Kunio AOKI ${ }^{3)}$ \\ ${ }^{2)}$ Fujita Health University, Japan, and ${ }^{3)}$ Aichi Cancer Center, Japan
}

\begin{abstract}
The effects of smoking on the function of frontal lobe in middle- and elderlyJapanese community dwellers were examined. The participants were 477 healthy individuals aged from 40 to 86 years old (the mean was 63 years old). The letter fluency test to measure verbal executive function and the D-CAT to measure information processing speed and sustained attention were used as the measures of cognitive function which reflects prefrontal cortex function. Analyses including age and years of education as covariates indicated that the intensity of smoking tended to influence digit cancellation performances and letter fluency performance. These findings suggest that smoking may exert a deleterious long-term effect on cognitive function.
\end{abstract}

Key words: smoking, attention, verbal fluency, function of frontal lobe

The examination of the smoking effects on cognitive function in later life of individuals has become one of the most popular topics of neuropsychological studies. This is due to the claim that cigarette use influence the cognition of elderly people and might be a risk factor of Alzheimer's disease (Fratiglioni, Ahlbom, Vitanen, \& Winbald, 1993; Lee, 1994).

Recent reports demonstrated that smoking causes structural changes in the human brain (Longstreth et al., 2001; Swan et al., 2000). Longstreth et al. (2001) underwent MRI to measure white matter, ventricles and sulcal size and found that factors such as smoking were associated with atrophy (enlarged ventricles and sulci) as well as age, sex, hypertension internal carotid artery wall thickness. Swan et al. (2000) measured white matter volumes by MRI for elderly men free from severe cognitive impairments. They determined the relationships between the levels of atrophy defined by low total brain volume and high white matter hyper-intensity volume and number of years smoking experience.

The study is the part of a joint cohort study between Yakumo Town and Nagoya University Medical School. Present authors are much indepted to staff members of Silver plaza in Yakumo Town for their help in data collection. Part of this study was supported by grants-in-Aid for scientific Research (No. 14201010) from the Ministry of Education, Science, Sports, and Culture of Japan to the first author.

Correspondence concerning this article should be addressed to Takeshi Hatta, Department of Psychology, Graduate School of Environmental Studies, Nagoya University, Furoh-cho, Chikusa-ku, Nagoya City 4648601, Japan (e-mail: thatta@info.human.nagoya-u.ac.jp). 
As apparent from the above short review, the effects of smoking on brain structural changes have been clarified, however empirical behavioral evidence of the smoking effect on cognitive function seems to be equivocal. For example, Ernst, Heishman, Spurgeon, and London (2001) examined the effects of smoking on visual attention (2 letter search tasks), verbal information processing (logical reasoning), and working memory (n-back tasks). They reported that logical reasoning showed no effect, while working memory and attention may have negative effects (deterioration of cognitive function). Hill, Nilsson, Nyberg, and Backman (2003) reported a cohort study in Sweden and found current smokers performed more poorly than non-smokers on the more cognitive demanding tasks such as Block design and free recall.

On the other hand, there are several reports that smoking has no substantial effect on cognition. Sakurai and Kanazawa (2002) examined the smoking effect on Buschke's selective reminding tests, a mental arithmetic task and a letter fluency task and concluded that a daily dose of nicotine had little effect on the performance of related memory, calculation and mental association. Edelstein, Kritz-Silverstein, and Barrett-Connor (1998) examined the effects of smoking on long-term memory and visual production tests and also concluded that the results offer no compelling evidence that cigarette smoking causes or prevents impaired cognitive function in old age. Hill (1989) reported that residual effects of smoking on problem solving, psychomotor speed, memory, attention span, perception and language production and showed no difference between non-smokers and ex-smokers on any measures, though smokers showed decrements only on psychomotor speed.

Then, the purpose of this study was to clarify the interactive relationship between smoking and cognitive function more precisely. As a cognitive function consisted of functional contribution of various brain parts, in this preliminary study, we focused on the prefrontal lobe function and examined the relations with behavioral measures in middleage and elderly community dwellers.

A cumulative record of research on cognitive aging shows that age-related cognitive declines are due to age-related alteration of brain structure (Raz, 1996). Therefore, it is natural to assume that there is an age-sensitive cognitive processing which is maintained by age-sensitive or vulnerable to aging areas of the brain. Based on the review, Raz (1996) suggested that brain structures that evolved in more ancient species and those that mature early in the course of human development, (i.e., later developed structures in both phylogenetic and ontogenetic aspects) such as globus pallidus, paleocerebellum, sensory cortices, and pons) are less affected by aging than the neocerebellar vermis, the neostriatum, and the association cortex, especially prefrontal cortex. This means that the prefrontal cortex is highly vulnerable to aging, the hippocampus is moderately vulnerable, the occipital cortex is mildly vulnerable and the pons is invariant regions of the brain. This proposal also suggests that effects of smoking on cognitive function must appear in cognitive function, especially, which strongly relates to prefrontal cortex regions.

In this study, we focused on the effect of smoking on attention and verbal fluency, because both attention and verbal function play the most fundamental roles among a wide range of human cognition. It is regarded that attention is a major psychological construct 
and most strongly related to prefrontal cortex function. It is suggested that the investigation of selective and focused attention must reveal the effects of smoking on prefrontal brain function. In other words, by using a sensitive behavioral test, which focus on the evaluation of selective and focused attention, the relationships between smoking and attention/verbal function can be demonstrated.

The D-CAT is regarded as one of the most sensitive behavioral tests for attention. The D-CAT (digit cancellation test) was developed to measure selective and focused attention (Hatta, Ito, \& Yoshizaki, 2001, 2004). The D-CAT, attention test, evaluates the following three levels; focused attention, sustained attention or concentration and selective attention, according to the attention model by Sohlberg and Mateer (1989) described later. In our study, word retrieving ability was assessed by the Japanese letter fluency test (Japanese version by Ito \& Hatta, 2001, 2004).

More concretely, we examined the effects of cigarette use on D-CAT tests for attention evaluation and on Japanese letter fluency test for word retrieving function in healthy middle age and elderly community dwellers.

\section{METHOD}

\section{Participants:}

Eight hundred and ninety five healthy rural community dwellers participated in this experiment. The data was based on an ongoing collaborative cohort project between Yakumo town (a rural town in Hokkaido Island) and Nagoya University Medical School and Fujita Medical University. The data used in this study was collected in August, 2002. All participants completed a health questionnaire which included not only the smoking condition in daily life but also other various daily life conditions such as drinking, food, physical exercise, etc. However, participants joined voluntarily to the neuropsychological examination, therefore, only the data of the participants who administered both the daily life questionnaire and the neuropsychological examination were analyzed. The participants who showed a mild dementia and any other signs of physical disease were deleted from the data analysis. The mean age of the participants used was 63 years and ranged from 40 to 86 years. Of the participants, 189 were male and 288 were female. All were healthy elderly people without signs of mild dementia or other neurological defects (this evaluation was based on the Clock Drawing test, immediate and delayed sentence memories, prospective memory, Stroop test, Money road test, and the QOL questionnaire).

\section{Smoking Cigarette:}

Followed by Schinka et al. (2002), intensity of smoking was used in the analyses. The intensity of smoking was calculated as the maximum amounts consumed over a sustained (minimum of 1 year) period of time. For smoking, it was calculated as the number of cigarettes per day. Participants were classified into 4 classes, non-smoker, light-smoker (mean is 9 cigarettes per day), medium-smoker (mean is 19 cigarettes per day), and heavy-smoker (mean is 26 cigarettes per day). The scientific background, such as nicotine influence on physical function, of this 4-types classification in smoking group is not clear, however it has been used in several studies (Schinka, Belanger, Mortimer, \& Graves, 2003). The number of participants consisting of the four groups was as follows; non-smoker group was 295, light-smoker group was 52 , medium-smoker group was 81 and heavy-smoker group was 49 as shown in Table 1.

\section{Cognitive Tests:}

In this study, attention was evaluated as the representative of the cognitive function by the Digit Cancellation Test (D-CAT) (Hatta et al., 2001). The Digit Cancellation Test (D-CAT) follows the attention model proposed by Sohlberg and Mateer (1989). They described a model of attention with five components 
(focused attention, sustained attention, selective attention, alternating attention, and divided attention) based on experimental and clinical research. Their model has hierarchical characteristics; each component of the hierarchy is viewed as requiring the effective functioning of the one below it. In their model, five hierarchical components cannot be discriminated easily into one of those more theoretical components. As such, an attention consists of several different factors. Working memory is also commonly included to hold and manipulate information in mind. The D-CAT aimed to evaluate first three levels of attention, focused attention, sustained attention concentration and selective attention.

The examiners administered the D-CAT for participants individually as a part of the neuropsychological examination of the Yakumo project. In the D-CAT, participants were requested to search the given target digit(s) (1 or 3 digits) on the randomly arranged digit sequences sheet, and scan the target digit(s) for 60 s. Two indices of Total Performance score, and Miss-ratio were evaluated. The Total Performance score refers to the total number of digits that the participant could scan (not the numbers of searched target digits but total range of digits searched), and assesses information processing speed, focused attention, and sustained attention (Hatta et al., 2001, 2004). The Miss-ratio score is also one of the indices of the D-CAT and it mainly reflects components such as sustained attention and selective attention.

To evaluate verbal fluency, a letter fluency test was administered individually. Participants were asked to generate Japanese nouns that begin with each letter, $/ \mathrm{a} /, / \mathrm{ka} /$, and $/ \mathrm{shi} /$, for $60 \mathrm{~s}$ in each letter. The total amount of generated nouns of those letters was measured.

\section{RESULTS}

Table 1 shows the demographic characteristics and the results of D-CAT and letter fluency as a function of four smoking groups.

According to the same criteria as Schinka et al. (2002), participants were classified into 4 classes, non-smoker, light-smoker (mean is 9 cigarettes per day), medium-smoker (mean is 19 cigarettes per day), and heavy-smoker (mean is 26 cigarettes per day). This index relates to the intensity of smoking and was calculated as the maximum amounts consumed over a sustained (minimum of 1 year) period of time but not the lifetime dose.

Age at the time of the study and number of years of formal education served as

Table 1. Demographic Characteristics and Cognitive Performance Statistics for Smoking Groups (SDs are shown in the parentheses.)

\begin{tabular}{lccccccccc}
\hline Group characteristics Score & \multicolumn{2}{c}{ Abstinent } & \multicolumn{2}{c}{ Light smokers } & Medium smokers & \multicolumn{2}{c}{ Heavy smokers } \\
\hline$N$ & \multicolumn{2}{c}{295} & \multicolumn{2}{c}{52} & \multicolumn{2}{c}{81} & \multicolumn{2}{c}{49} \\
Age & 63.1 & $(9.2)$ & 60.7 & $(11.0)$ & 61.4 & $(9.9)$ & 63.7 & $(9.5)$ \\
Years of education & 10.4 & $(2.3)$ & 7.0 & $(2.1)$ & 7.7 & $(2.3)$ & 7.6 & $(2.6)$ \\
Cigarettes per day & & 0 & & 9 & & 19 & & 36 \\
Letter fluency & 19.9 & $(7.0)$ & 23.5 & $(8.0)$ & 19.7 & $(7.9)$ & 18.1 & $(6.9)$ \\
TP-1(D-CAT) & 250.7 & $(65.2)$ & 259.9 & $(61.5)$ & 261.8 & $(70.3)$ & 236.9 & $(61.9)$ \\
TP-3(D-CAT) & 156.9 & $(39.3)$ & 158.7 & $(39.4)$ & 152.6 & $(41.5)$ & 143.3 & $(35.3)$ \\
Miss-1(D-CAT) & 0.06 & $(0.08)$ & 0.03 & $(0.05)$ & 0.052 & $(0.08)$ & 0.05 & $(0.06)$ \\
Miss-3(D-CAT) & 0.13 & $(0.1)$ & 0.096 & $(0.07)$ & 0.12 & $(0.09)$ & 0.12 & $(0.12)$ \\
\hline
\end{tabular}


control variables. For one set of analysis, intensity of smoking was used as covariate control variables.

An analysis of covariance (ANCOVA) was conducted with groups determined by smoking indices to examine the impact of intensity of smoking on cognitive function. Control variables served as covariates for the analyses. As similar to Schinka et al. (2002), ANCOVA did not include sex as an independent variable, because of the substantial loss of statistical power.

Results of the ANCOVA on cognitive ability with the effect of smoking intensity are presented in Table 2. From the analyses of smoking intensity, the years of education were found to be a significant $(p<.05)$ covariate for the letter fluency, all indices on D-CAT. Smoking intensity was also found to be significantly covariate for letter fluency and TP 3 (total performance in three digits cancellation task) in the D-CAT.

As seen from Table 1, the performance of light-smokers was the best among the four groups for the letter fluency test and TP3 index on the D-CAT. In the D-CAT, several indices can be analyzed to examine the attention function of the participant. However, in this study, only TP1 and TP3 were employed because of parsimony, that is, to simplify the aim of the study. According to D-CAT manual, TP1 (cancel one target digit as fast as possible) and TP3 (cancel three target digits as fast as possible) involve different psychological factors. It is assumed that TP3 reflects working memory factors more strongly than of TP1.

Table 2. Results of ANCOVA

\begin{tabular}{llccc}
\hline Letter fluency & & $F$ value & Probability & $\eta 2$ \\
\hline \multirow{5}{*}{ TP1(D-CAT) } & Age & 2.696 & 0.101 & 0.006 \\
& Education & 30.444 & 0.000 & 0.061 \\
& Smoking & 3.667 & 0.012 & 0.023 \\
& Age & 101.213 & 0.000 & 0.177 \\
& Education & 40.105 & 0.000 & 0.078 \\
TP3(D-CAT) & Smoking & 0.897 & 0.442 & 0.006 \\
& & & & \\
& Age & 58.340 & 0.000 & 0.110 \\
& Education & 33.988 & 0.000 & 0.067 \\
& Smoking & 3.43 & 0.017 & 0.021 \\
Miss 1(D-CAT) & & & & \\
& Age & 14.316 & 0.000 & 0.029 \\
& Education & 5.641 & 0.018 & 0.012 \\
& Smoking & 0.793 & 0.498 & 0.005 \\
Miss 3(D-CAT) & & & & \\
& Age & 25.421 & 0.000 & 0.051 \\
& Education & 4.4968 & 0.034 & 0.009 \\
& Smoking & 1.157 & 0.326 & 0.007 \\
\hline
\end{tabular}




\section{DISCUSSION}

Why do people smoke? They claim that smoking enhances cognitive performance, smoking makes one relax, smoking is necessary to be adult-like, etc. The background of these claims are sometimes supported and sometimes not supported by experimental studies. For example, Lawrence, Ross, and Stein (2002) reported a fMRI study where smokers were given a sustained attention task (rapid visual information processing task). Their data suggested that nicotine improves attention in smokers by enhancing activation in areas known to be associated with visual attention, arousal and motor activation. Ghatan et al. (1998) also reported a regional cerebral blood flow (rCBF) study. They found that nicotine induced $\mathrm{rCBF}$ decreases in the anterior cingulate cortex and cerebellum in smokers, and concomitant increase in the occipital cortex. That is, special changes were induced in areas pertaining to the anterior attention system and to higher order visual cortex. On the other hand, a recent physiological study by Ascioglu, Dolu, Golgeli, Suer, and Ozesmi (2004) showed that cigarette smoking does not improve cognitive processing, where they measured ERPs and EPs on standard oddball paradigm performance. Herbert, Foulds, and Fife-Schaw (2001) examined whether smoking reduces anxiety. Participants were either allowed to smoke normally or not allowed to smoke and were given a computer-made rapid visual information processing (RVIP) task. Mood was measured by both State Anxiety Inventory (SAI) and Feeling state Questionnaire (FSQ) before and immediately after the RVIP. Smoking did not decrease anxiety or increase RVIP performance compared with not smoking.

The purpose of this preliminary study was to clarify the effects of cigarette smoking on prefrontal lobe functions. As described earlier, the findings of smoking effects on cognitive function are equivocal. Some found substantial effect and others showed no substantial effects on cognitive function.

In this study, we focused on two tests, letter fluency test and D-CAT, which are regarded as tests for prefrontal cortex function. There is ample evidence to suggest a strong relationship between the prefrontal cortex and attention (especially focused attention and sustained attention or working memory) and word retrieval. Functional neuro-imaging studies of intact adults have shown that the prefrontal cortex is activated during the performance of sustained attention or working memory (e.g., Awh et al., 1996; Fiez, Raichle, Balota, Tallal, \& Petersen, 1996; Jonides et al., 1993; Jonides, Smith, Marshuetz, Koeppe, \& Reuter-Lorenz, 1998; Paulesu, Frith, \& Frackwiak, 1993; Smith et al., 1995). A fMRI study by Mead et al. (2002) also indicated that the component of selective attention (such as the Stroop test) invites the activation of frontal cortex, especially around the area of BA44.

The findings of this study demonstrated that smoking covariates prefrontal lobe performance, especially on the letter fluency test and TP3 index on the D-CAT. As the index of TP3 in the D-CAT seems to reflect information processing speed and sustained attention, our findings might suggest the substantial effect of long term smoking on processing speed and sustained attention abilities. Table 1 shows the fact that heavysmokers who generally have a long history of smoking were the worse in TP3 
performance. Our findings seems to coincident with the study by Ernst et al. (2001) which showed smoking effects on visual attention (2 letter search task) and working memory but not on verbal information processing (logical reasoning task), and Hill (1989) who reported that residual effects of smoking showed on a psychomotor speed task but not on memory, attention span, perception. Ghatan et al. (1998) suggested that brain activity in areas pertaining to the anterior attention system is activated by nicotine infusion. The present findings seem to suggest that attention related prefrontal function is vulnerable to smoking. However, our results did not necessarily support the claim that cigarette smoking gave wrong after effects on prefrontal function because light-smokers showed good behavioral performances. Further systematic examinations have to be prepared.

We have to mention the possible reasons why light-smokers showed good behavioral performances. Does our results recommend small amount of smoking to sustain prefrontal lobe function? It is definitely too early conclusion. As mentioned earlier, the present criterion of grouping smokers based on the intensity but not to the lifetime dose. There are other several possible reasons why light-smokers showed good performances in our study. One is that in the light-smokers group involve participants who have once smoked in early age but now is abstinent. This type of participants might be small in the moderate and heavy smokers group. There must be many intelligent participants or holders of good prefrontal lobe function who were informed negative campaign that cigarette smoking invites health problems such as bronchitis, bronchial asthma, lung cancer and are trying refrain smoking but not yet become abstinent. Further more precise examination of the validity of group classification criterion should be administered. The other is that abstinent involve more females that males. Possible sex difference on the attention and verbal fluency might be contaminated in the present study. This interpretation might invite discussion the contribution of the effects of well-known female advantage on verbal function. Recent finding indicate that well known female advantage over male in verbal abilities diminish after menopause (Hatta et al., 2005).

In this study, we focused only on the effects of smoking on prefrontal lobe function, however, smokers used to be drinkers. Recent reports showed that heavy drinking is associated with brain atrophy (Kubota et al., 2001; Mukamal, Longstreth, Mittelman, Crum, \& Siscovick, 2001). Kubota et al. (2001) measured frontal lobe shrinkage on MRI of four classes of people (abstainers, light, moderate and heavy drinkers) and found that although people of light to moderate alcohol consumption showed no increase in the rate of frontal lobe shrinkage, heavy-drinkers (more than $418.1 \mathrm{~g}$ ethanol per week) showed a significant shrinkage compared with abstainers. Mukamal et al. (2001) also reported similar findings. As described before, attention and verbal fluency are regarded as the most sensitive functions of the prefrontal cortex, we wish to extend our examination of smoking effects on prefrontal lobe function in the case of concurrent alcohol drinking with smoking. Therefore, we should note, from the scientific points of view, that the smoking effects on prefrontal function remains unclear. Further systematic examination should be prepared. 


\section{REFERENCES}

Ascioglu, M., Dolu, N., Golgeli, A., Suer, C., \& Ozesmi, C. 2004. Effects of cigarette smoking on cognitive processing. International Journal of Neuroscience, 114, 381-390.

Awh, E., Jonides, J., Smith, E. E., Schumacher, E. H., Koeppe, R. A., \& Katz, S. 1996. Dissociation of storage and rehearsal $\mathrm{n}$ verbal working memory: Evidence from positron emission tomography. Psychological Science, 7, 25-31.

Edelstein, S. L., Kritz-Silverstein, D., \& Barrett-Connor, E. 1998. Prospective association of smoking and alcohol use with cognitive function in an elderly cohort. Journal of Wemen's Health, 7, 1271-1281.

Ernst, M., Heishman, S. J., Spurgeon, L., \& London, E. D. 2001. Smoking history and nicotine effects on cognitive performance. Neuropsychopharmacology, 25, 313-319.

Fiez, J. A., Raichle, M. E., Balota, D. A., Tallal, P., \& Petersen, S. E. 1996. PET activation of posterior temporal regions during auditory word presentation and verb generation. Cerebral Cortex, 6, 1-10.

Fratiglioni, L., Ahlbom, A., Vitanen, M., \& Winbald, B. 1993. Risk factors for late-onset Alzheimer's disease: A population-based. Case-control study. Annuals of Neurology, 33, 258-266.

Ghatan, P. H., Ingvar, M., Eriksson, L., Stone-Elander, S., Serrander, M., Elberg, K. et al. 1998. Cerebral effects of nicotine during cognition in smokers and non-smokers. Psychopharmacology, 136, 179189.

Hatta, T., Ito, Y., \& Yoshizaki, K. 2001. Digit cancellation test (D-CAT) for attention. Osaka: Union Press.

Hatta, T., Ito, Y., \& Yoshizaki, K. 2004. Digit Cancellation Test (D-CAT): A new screening test for attention. Manuscript submitted for publicaiton.

Hatta, T., Nagaya, K., Onishi, M., Iwahara, A., Nagahara, N., \& Ito, E. 2005. Age related sex difference in higher cognitive abilities of healthy middle and old age people. Proceedings of the International Behavioral Development Symposium at Minot State University, 41.

Herbert, M., Foulds, J., \& Fife-Schaw, C. 2001. No effect of cigarette smoking on attention or mood in nondeproved smokers. Addiction, 96, 1349-1356.

Hill, R. D. 1989. Residual effects of cigarette smoking on cognitive performance in normal aging. Psychology and Aging, 4, 251-254.

Hill, R. D., Nilsson, L. G., Nyberg, L., \& Backman, L. 2003. Cigarette smoking and cognitive performance in healthy Swedish adults. Age and Ageing, 32, 548-550.

Ito, E., \& Hatta, T. 2001. Development of the verbal fluency test for Japanese. Studies in Informatics and Sciences, 15, 81-96.

Ito, E., \& Hatta, T. 2004. Performance of verbal fluency tasks in Japanese healthy adults - effect of gender, age and education on the performance - Japanese Journal of Neuropsychology, 20, 254-263.

Jonides, J., Smith, E. E., Koeppe, R. A., Awh, E., Minoshima, S., \& Mintun, M. A. 1993. Spatial working memory in humans as revealed by PET. Nature, 363, 623-625.

Jonides, J., Smith, E. E., Marshuetz, C., Koeppe, R. A., \& Reuter-Lorenz, P. A. 1998. Inhibition in verbal working memory revealed by brain activation. Proceedings of the National Academy of Sciences of the United States of America, 95, 8410-8413.

Kubota, M., Nakazaki, S., Hirai, S., Saeki, N., Yamamura, A., \& Kusaka, T. 2001. Alcohol consumption and frontal lobe shirinkage: Study of 1432 non-alcoholic subjects. Journal of Neurosurgery and Psychiatry, 71, 104-106.

Lawrence, N. S., Ross, T. J., \& Stein, E. A. 2002. Cognitive mechanisms of nicotine on visual attention. Neuron, 36, 539-548.

Lee, P. N. 1994. Smoking and Alzheimer's disease: A review of the epidemiological evidence. Neuroepidemiology, 143, 219-227.

Longstreth, W. T., Diehr, P., Manolio, T. A., Beauchamp, N. J., Jungreis, C. A., \& Lefkowitz, D. 2001. Cluster analysis and patterns of findings on cranial magnetic resonance imaging of the elderly: The cardiovascular health study. Archives of neurology, 58, 635-640.

Mead, L. A., Mayer, A. R., Bobholz, J. A., Woodley, S. J., Cunningham, J. M., Hammeke, T. A. et al. 2002. Neural basis of the Stroop interference task: Response competition or selective attention. Journal of the International Neuropsychological Society, 8, 735-742.

Mukamal, K. J., Longstreth, W. T., Mittelman,M. A., Crum, R. M., \& Siscovick, D. S. 2001. Alcohol consumption and subclinical findings on magnetic resonance imaging of the brain in older adults: The 
cardiovascular health study. Stroke, 32, 1939-1946.

Paulesu, E., Frith, C. D., \& Frackwiak, R. S. J. 1993. The neural correlates of the verbal component of working memory. Nature, 362, 342-345.

Raz, N. 1996. Aging of the brain and its impact on cognitive performance: Integration of structural and functional findings. In F. I. M. Craik \& T. A. Salthouse (Eds.), The handbook of aging and cognition (pp. 1-90). NJ: LEA.

Sakurai, Y., \& Kanazawa, I. 2002. Acute effects of cigarettes in non-deprived smokers on memory, calculation and execute functions. Human Psychopharmacology, 17, 369-373.

Schinka, J., Belanger, H., Mortimer, J. A., \& Graves, A. B. 2003. Effects of the use of alcohol and cigarettes on cognition in elderly African American adults. Journal of the International Neuropsychological Society, 9, 690-697.

Schinka, J., Vanderploeg, R. D., Rogish, M., Graves, A. B., Mortimer, J. A., \& Ordorica, P. I. 2002. Effects of the use of alcohol and cigarettes on cognition in elderly adults. Journal of the International Neuropsychological Society, 8, 811-818.

Smith, E. E., Jonides, J., Koeppe, R. A., Awh, E., Schumacher, E. H., \& Minoshima, S. 1995. Spatial versus object working memory: PET investigation. Journal of Cognitive Neuroscience, 7, 337-356.

Sohlberg, M. M., \& Mateer, C. A. 1989. Introduction to cognitive rehabilitation: Theory and practice. New York: Oxford University press.

Swan, G. E., De Carli, C., Miller, B. L., Reed, T., Wolf, P. A., \& Carmelli, D. 2000. Biobehavioral characteristics of nondemented older adults with subclinical brain atrophy. Neurology, 54, 21082114.

(Manuscript received May 28, 2004; Revision accepted October 4, 2005) 